\title{
Probability of US heat waves affected by a subseasonal planetary wave pattern
}

\author{
Haiyan Teng ${ }^{1 \star}$, Grant Branstator ${ }^{1}$, Hailan Wang ${ }^{2}$, Gerald A. Meehl' and Warren M. Washington ${ }^{1}$
}

\begin{abstract}
Heat waves are thought to result from subseasonal atmospheric variability. Atmospheric phenomena driven by tropical convection, such as the Asian monsoon, have been considered potential sources of predictability on subseasonal timescales. Mid-latitude atmospheric dynamics have been considered too chaotic to allow significant prediction skill of lead times beyond the typical 10-day range of weather forecasts. Here we use a 12,000-year integration of an atmospheric general circulation model to identify a pattern of subseasonal atmospheric variability that can help improve forecast skill for heat waves in the United States. We find that heat waves tend to be preceded by 15-20 days by a pattern of anomalous atmospheric planetary waves with a wavenumber of 5 . This circulation pattern can arise as a result of internal atmospheric dynamics and is not necessarily linked to tropical heating. We conclude that some mid-latitude circulation anomalies that increase the probability of heat waves are predictable beyond the typical weather forecast range.
\end{abstract}

T he increasing severity of heat waves in recent decades and projections of even more intense and frequent heat waves in the future $e^{1-3}$ has resulted in a growing demand for skillful predictions of these high-impact events beyond the 10-day forecast range. On the subseasonal timescale, low-frequency intraseasonal tropical phenomena such as the Madden-Julian oscillation ${ }^{4,5}$, fluctuations of Asian monsoon precipitation and slowly varying boundary conditions are commonly regarded as the primary potential sources of predictability. The mid-latitude circulation, however, is dominated by weather noise and its evolution is strongly chaotic making it unpredictable after about 10 days for typical initial conditions. However, this 10-day limit of prediction skill does not exclude the possibility that some circulation states can be substantially more predictable than the average $e^{6,7}$, either from having large amplitude or being associated with patterns that are intrinsically of low frequency.

Some case studies ${ }^{8-10}$ have suggested that US heat waves (and associated droughts) may be preceded and accompanied by quasi-stationary large-scale mid-latitude atmospheric Rossby waves. However, the scarcity of extreme events in the observational records together with the inherent noisiness of mid-latitude weather fluctuations make it difficult to test this possibility. To avoid this problem here we use a 12,000-year simulation from an atmospheric general circulation model (GCM), called Community Atmospheric Model version 3 (CAM3), so that there are sufficient samples to examine these unusual events. In this simulation monthly varying sea surface temperature (SST) is prescribed to have present-day climatological values. As a result, atmospheric fluctuations are mainly produced by internal atmospheric dynamics.

\section{US heat wave statistics}

We devote our attention to events in which surface air temperature (SAT) is persistently high somewhere over the continental United States. Specifically, a day during June-August is considered to be a heat wave day if on that day and each of four succeeding days there are more than ten grid points (corresponding to $5 \%$ of the searched domain) over the US continental area within the domain of $125^{\circ} \mathrm{W}-70^{\circ} \mathrm{W}, 25^{\circ} \mathrm{N}-50^{\circ} \mathrm{N}$ with daily averaged SAT exceeding a threshold value, and the centre of these warm points does not move faster than $5^{\circ}$ latitude or longitude per day. The temperature threshold we use varies with grid point as well as day of the year. It corresponds to the 97.5 percentile for historical daily temperatures within a 15-day window centred on the day of the year of the potential heat wave day. Consecutive heat wave days are grouped into a single heat wave event.

We also apply the same procedure to the National Centers for Atmospheric Prediction/National Center for Atmospheric Research reanalysis ${ }^{11}$ (NCEP/NCAR reanalysis) during the period of 1948-2012. A comprehensive comparison of the model and the observations is difficult owing to the brevity of the observations. Nevertheless, there are qualitative similarities between the model and the reanalysis data regarding the life span, intensity and frequency of the heat waves (Fig. 1), which indicate that the basic characteristics of the simulated heat waves are realistic.

\section{A zonal wavenumber-5 precursor pattern}

To look at the temporal evolution of planetary waves associated with the heat waves in the model, we designate the first day of a heat wave event as day 0 and construct composites on each day from day -20 to day 5 using daily subseasonal anomalies of $300 \mathrm{hPa}$ streamfunction (Fig. 2). Subseasonal anomalies are daily departures from both the long-term mean for each day and the seasonal (June-August) mean for any particular year. To avoid contamination from previous heat wave events, we use only 2,300 events that have no heat wave days in the preceding 20 days. From day -20 to day 5 (Fig. 2), there is a wavenumber-5 structure slowly propagating westwards as highlighted by the yellow dashed lines, at a speed of roughly $2-3^{\circ}$ longitude per day. Both the spatial structure and the movement are reminiscent of atmospheric Rossby waves trapped in the midlatitude jet stream waveguide ${ }^{12,13}$. The wavenumber- 5 structure is equivalent barotropic, though the lower tropospheric circulation anomalies (figure not shown) over the Asian summer monsoon region are fairly weak.

On day 0, the entire continental United States and Mexico are dominated by anticyclonic circulation aloft and much reduced 

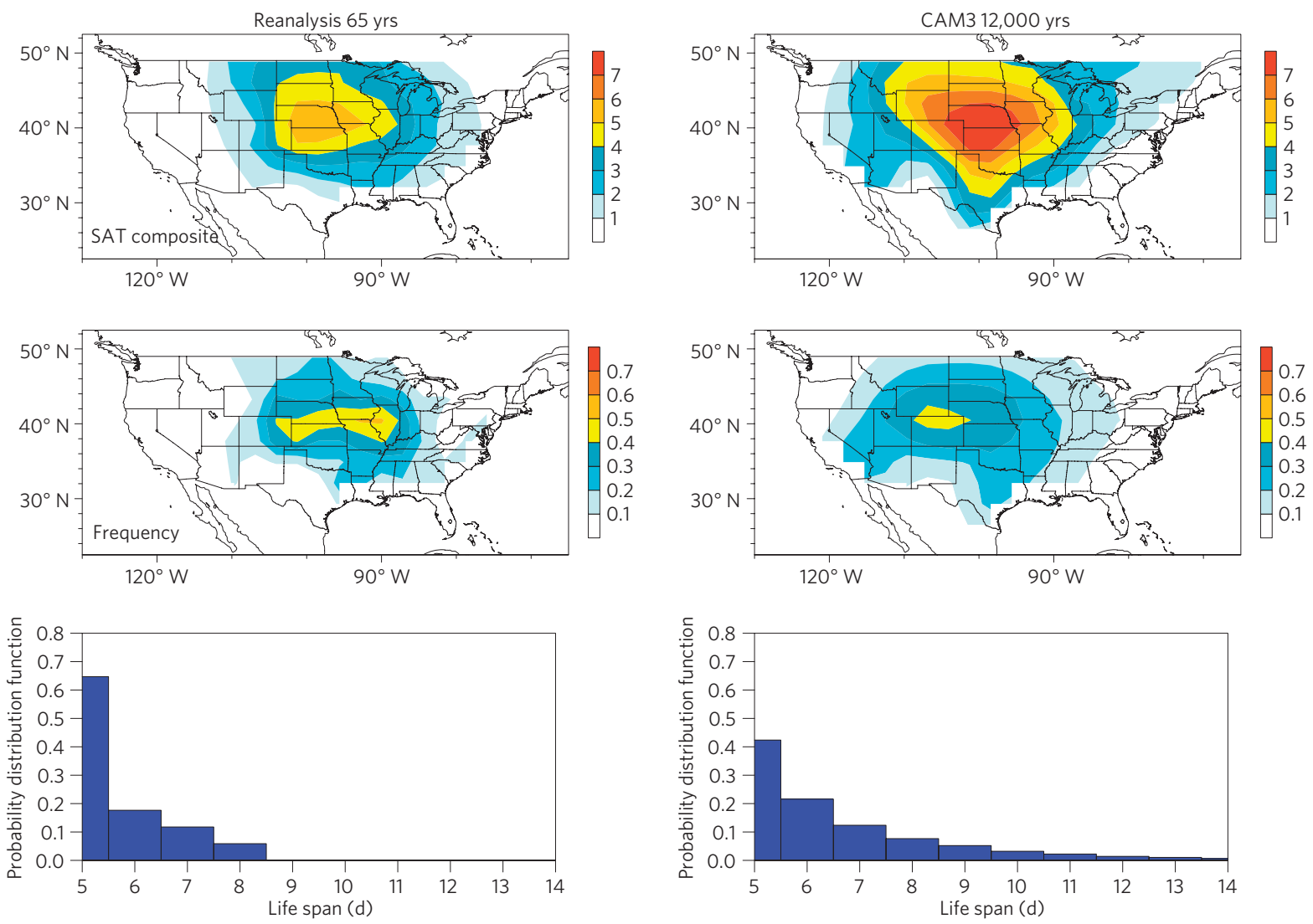

Figure 1 | Comparison of the model heat wave statistics with observations. Climatological SAT anomalies ( ${ }^{\circ} \mathrm{C}$; top panels) and frequency of occurrence (middle panels) of the US heat wave days and life span of the events (bottom panels) in the NCEP/NCAR reanalysis (left panels) and CAM3 simulation (right panels).

precipitation, which is consistent with observations of heat waves in nature ${ }^{3,14,15}$. Thereafter, the two positive streamfunction centres over the Bering Strait and the continental US disappear on day 5 and day 15 (not shown) respectively and the wavenumber-5 pattern fades away.

The Plumb flux ${ }^{16,17}$ has been frequently used as a diagnostic of the energy propagation of stationary atmospheric Rossby waves. It indicates (arrows in Fig. 2) that although the phase of the wavenumber-5 Rossby wave propagates slightly westwards from day -15 to day 0 , energy moves consistently eastwards and completes a global circle from day -10 to day 10 , which is roughly consistent with the group velocity of a stationary zonal wavenumber-5 disturbance trapped in the tropospheric jet ${ }^{18}$. Over the Pacific, there are also substantial similarities in both the Plumb fluxes and the Rossby wave with some observed heat wave events (for example, the 1980 heat wave/drought ${ }^{10}$ ) on about day 0 .

The strongest fluxes are located at the jet entrance and exit regions over both the Pacific and the North Atlantic oceans, suggesting that instabilities of the jet streams or interaction with transient eddies ${ }^{19}$ may be instrumental during the life cycle of the heat waves. That synoptic eddies may be contributing to maintenance is supported by a plot (Supplementary Fig. 3) of the streamfunction tendency from synoptic eddy vorticity fluxes. That plot shows that the anomalous tendencies have the same sign as the streamfunction anomalies during day -20 to day 5 in the two jet regions.

Although there is consistent change in precipitation (shading in Fig. 2) as the energy propagates eastwards from day -10 to day 5, there are no organized precipitation anomalies in either the tropics or in the Asian summer monsoon region around day -15 or earlier. Although SST is fixed in the experiment, the subseasonal variability of precipitation is comparable to that of the observations (Supplementary Fig. 1). Therefore, we do not think this lack of connection with the tropics is caused by any deficiency of tropical precipitation anomalies resulting from the simple treatment of SSTs. Instead, it further supports the interpretation that the wavenumber- 5 structure is produced by internal dynamics in the mid-latitudes, with submonthly transient vorticity flux anomalies playing an important role, similar to the findings in other studies ${ }^{8}$.

\section{Pattern of intrinsic subseasonal variability}

Unlike in the model simulation there are no statistically significant subseasonal precursor patterns in the reanalysis fields. This is probably because there are so few heat waves in the short observational record that the signal cannot be distinguished from noise with statistical certainty. However, a similar wavenumber-5 pattern has been noted in nature in boreal winter ${ }^{20}$ and summer ${ }^{21,22}$. It stands out as first empirical orthogonal function (EOF1) of the subseasonal variability of $300 \mathrm{hPa}$ streamfunction monthly anomalies in the reanalysis data (Fig. 3, top). As centres of action in EOF patterns do not necessarily co-vary, we calculate the coherency in daily reanalysis streamfunction time series from the five centres. This calculation shows significant coherency between any pair for periods longer than 20 days (figure not shown), an indication that the summer wavenumber- 5 pattern is likely to be a physical mode on subseasonal timescales.

In CAM3, the wavenumber-5 pattern is represented by EOF2, whereas EOF1 emphasizes a zonally symmetric component of variations in the circulation (Fig. 3, second and third panels). Pattern 


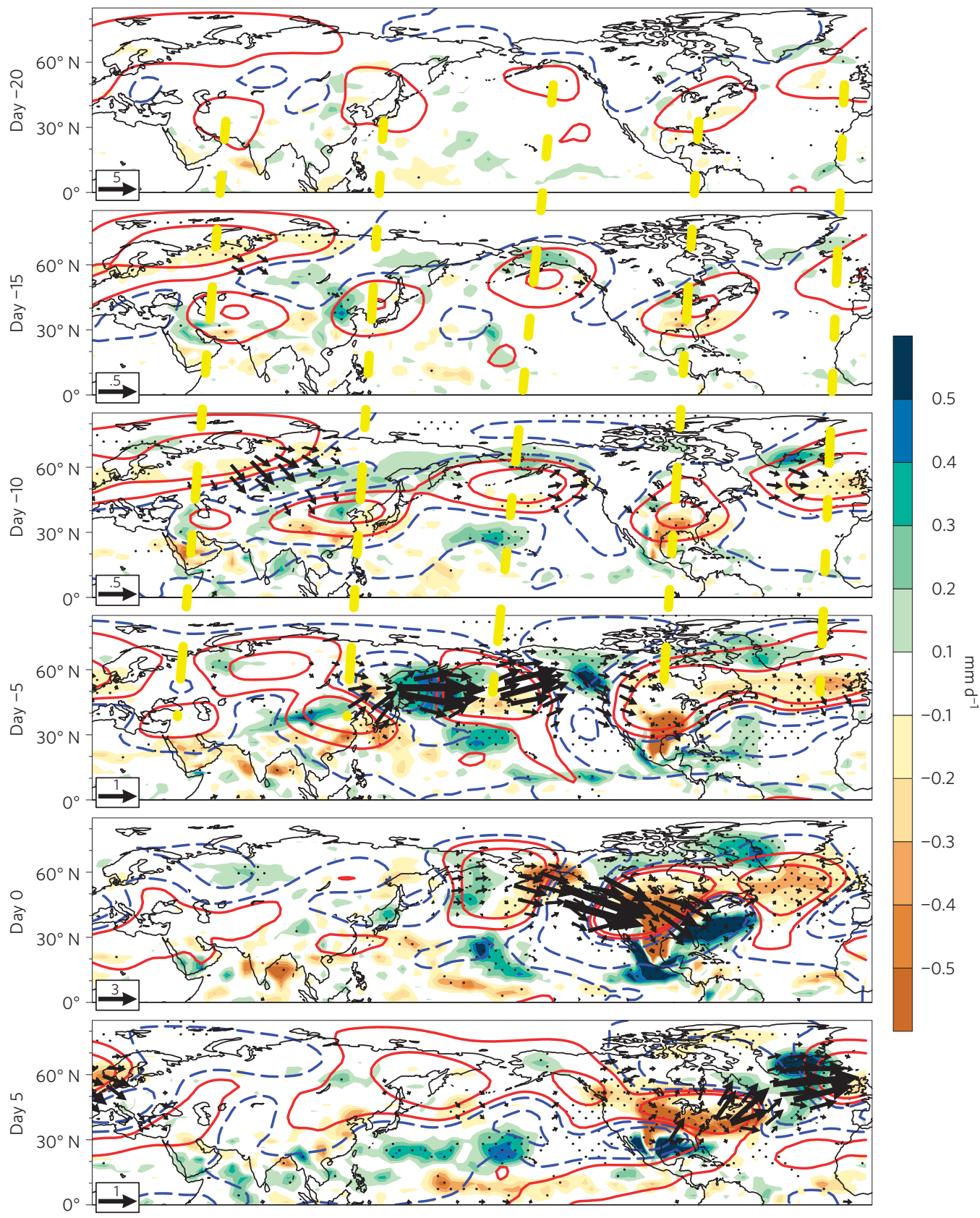

Figure 2 | Temporal evolution of the heat waves. Contours represent $300 \mathrm{hPa}$ streamfunction anomalies at $\pm 0.2, \pm 1.0 \times 10^{6} \mathrm{~m}^{2} \mathrm{~s}^{-1}$ levels $(\mathrm{most}$ areas with absolute values larger than $0.2 \times 10^{6} \mathrm{~m}^{2} \mathrm{~s}^{-1}$ are significant at the $95 \%$ level; see Supplementary Figs 4, 5 for significance tests). Shading represents composite precipitation $\left(\mathrm{mm} \mathrm{d}^{-1}\right)$, with stippling indicating the $95 \%$ significance level from Student's $t$-test, and arrows are the Plumb flux vectors with magnitudes larger than $0.1 \mathrm{~m}^{2} \mathrm{~s}^{-2}$. Dashed yellow lines in the top four panels highlight the position of the five mid-latitude high-pressure areas (the wavenumber-5 pattern) and their movement as a function of time.

correlations between EOF2 and the heat wave streamfunction composites (Fig. 2) are significant at the $99 \%$ level on each day from day -20 to day -12 based on a Monte Carlo test. EOF1 in the reanalysis seems to combine the characteristics of the leading two EOFs in CAM3.

The CAM3 EOF2, with its distinctive wavenumber-5 pattern, has a stronger connection with US heat waves than does EOF1. This is seen by two-dimensional probability distribution function (PDF) plots of SAT averaged over the continental United States within $125^{\circ} \mathrm{W}-70^{\circ} \mathrm{W}, 25^{\circ} \mathrm{N}-50^{\circ} \mathrm{N}$ versus projections of daily 300 hPa streamfunction anomalies onto EOF1 and EOF2 (Fig. 3 bottom middle and right panels). There is a clear linear relationship between the second principal component (PC2) and SAT when all days are considered. But for a given value of PC2, SAT is much stronger on heat wave days than it normally is, suggesting that factors other than PC2 must also contribute to the formation of the heat waves. Also presented in the bottom row of Fig. 3 is a scatter plot for reanalysis domain-averaged SAT and projections of daily $300 \mathrm{hPa}$ streamfuction anomalies onto the zonally asymmetric component of reanalysis EOF1. In this diagram the connection between wavenumber- 5 and SAT, including extreme events, is again present though not as clearly as in the large GCM data set.

Based on a strong connection between the strength of the Indian summer monsoon and the wavenumber- 5 pattern in nature, 
a
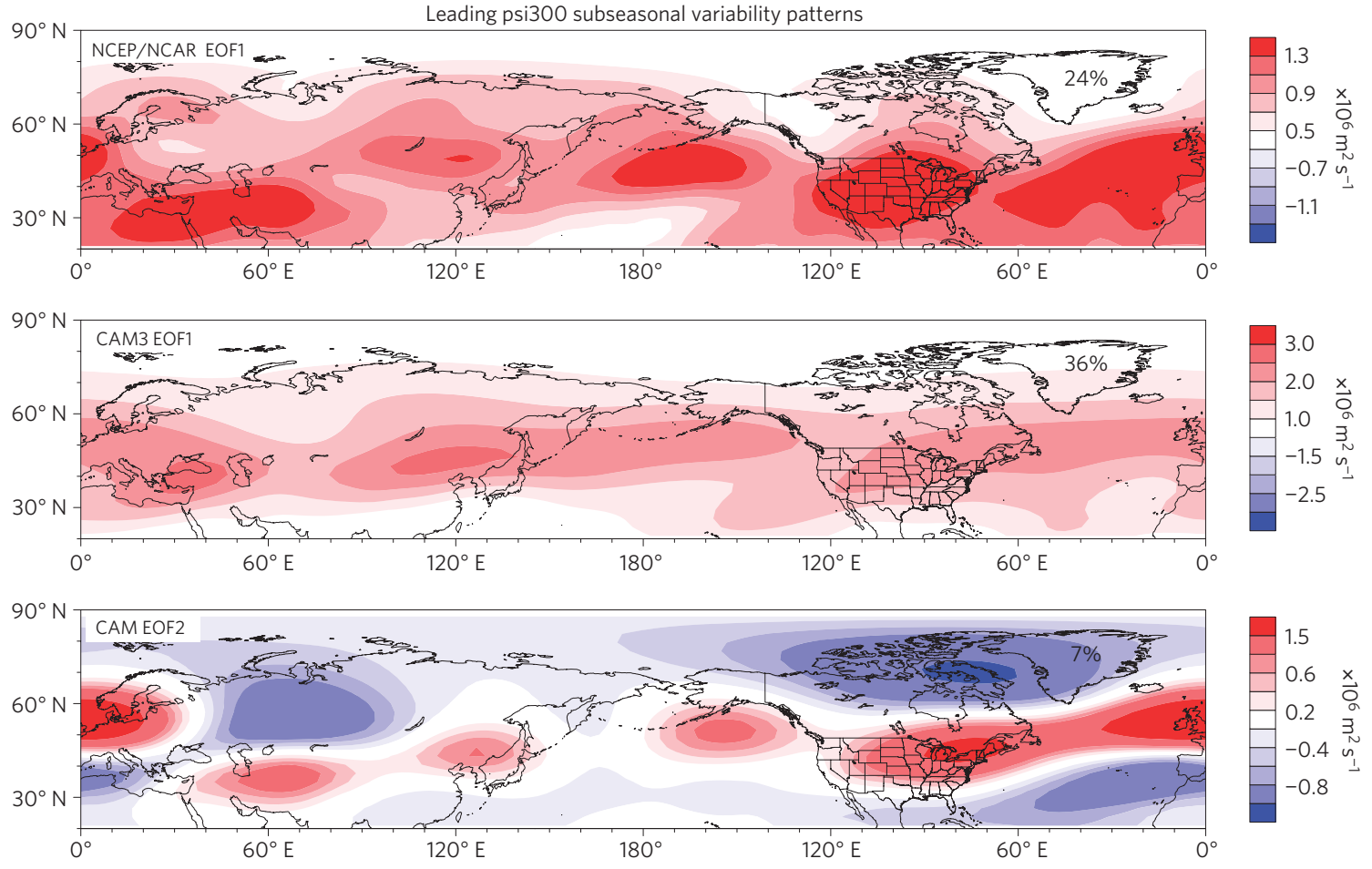

b

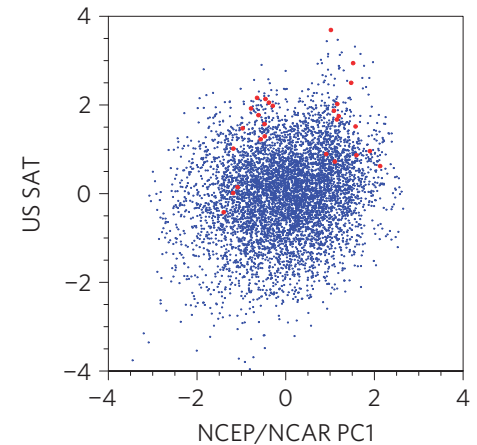

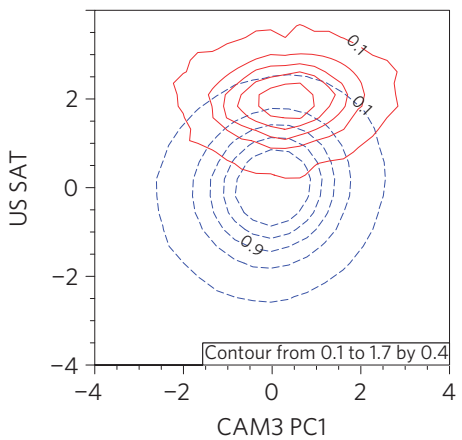

Figure $\mathbf{3}$ | Prominent subseasonal variability patterns. a, EOF1 of June-August subseasonal $300 \mathrm{hPa}$ streamfunction monthly anomalies in the reanalysis (top panel) and the leading two EOFs (middle and bottom) in CAM3. b. Shown is a scatter plot of daily US continental SAT versus projections of daily streamfunction subseasonal anomalies on the zonally asymmetric component of EOF1 in the reanalysis (left) and probability density functions (PDFs) of daily US continental SAT and streamfunction projections onto the two leading EOFs in CAM3 (middle and right) for all summer days (blue) and the heat wave days (red).

it has been hypothesized that an interaction with the Indian summer monsoon heat source is instrumental in maintaining the wavenumber-5 pattern ${ }^{21}$. For CAM3 we have examined PDFs of daily precipitation anomalies in both the Bay of Bengal and the western North Pacific, which are the key regions for the Asian summer monsoon. We find no distinct shifts in these PDFs during days when the wavenumber-5 pattern is either developing or at maturity. This suggests the monsoon precipitation is not key to producing the summer wavenumber-5 pattern in CAM3. Given the central role this pattern seems to play in initiating and maintaining US heat waves, this result is also consistent with our finding that tropical precipitation anomalies are not connected with heat wave events in this model. However, we cannot exclude the possibility that monsoon rainfall may initiate or enhance heat waves in fully coupled (atmosphere-ocean) experiments or in nature by stimulating or strengthening the wavenumber- 5 structure.

\section{Implication for subseasonal prediction of US heat waves}

The suggestion in Fig. 2 that wavenumber-5 anomalies tend to precede US heat waves by 15 days makes them potentially valuable for subseasonal prediction of these extreme events. To investigate this possibility further, we project seven-day running mean streamfunction anomalies onto the day -15 composite pattern shown in Fig. 2 (the precursor pattern) and calculate the fraction of cases that develop into heat waves 15 days later as a function of the strength of the projection. The conditional probability (red bars in Fig. 4) indicates the stronger the amplitude of a positive episode of the precursor pattern the greater the likelihood of a heat wave two weeks later. Without any precondition the probability of a randomly picked day being a heat wave day is about $1.5 \%$ (the number of heat wave days divided by the total number of summer days in the simulation, denoted as $P_{0}$ ). The figure indicates a one standard deviation projection onto the composite pattern and doubles the chances of a heat wave 15 days later; a two 


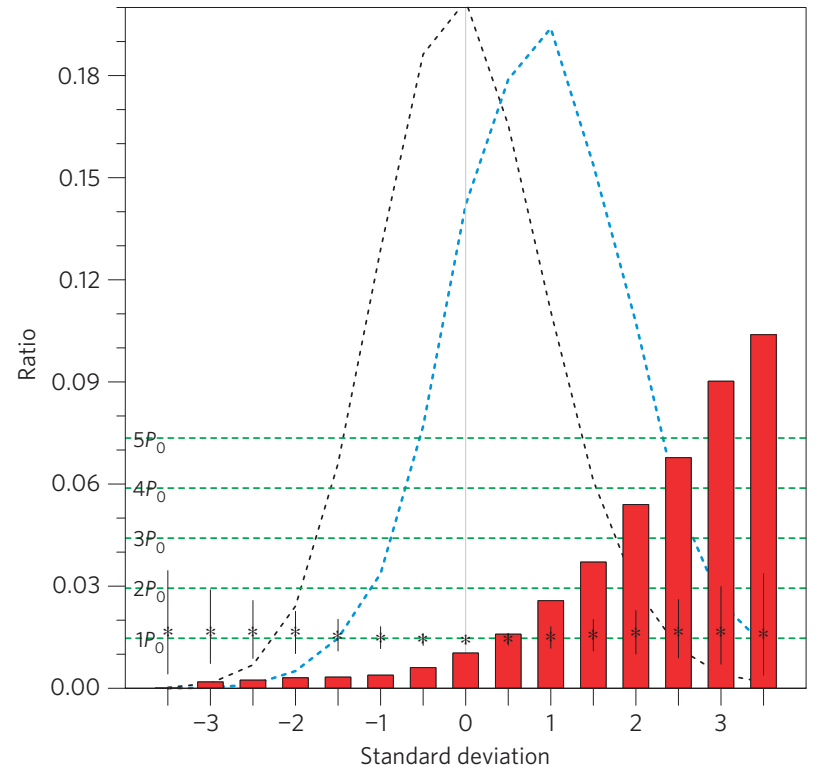

Figure 4 | Probability of the heat waves in CAM3 conditioned on the standardized amplitude of the wavenumber- 5 pattern 15 days earlier (red bars). The probability of the heat waves without any precondition $\left(P_{0}\right)$ is about 0.015 (the lowest green dashed line). Asterisks and black vertical lines represent the average and 5th to 95th percentile range, respectively, of the conditional probability of 1,000 random patterns of subseasonal variability. The black and blue dashed lines are the PDFs of amplitude of the wavenumber- 5 pattern in all summer days and on day -15 of the heat wave days, respectively.

standard deviation projection quadruples the chances compared with a randomly picked case. However, initial anomalies of -0.5 standard deviation reduce the chance of having a heat wave 15 days later by half. A Monte-Carlo-based significance test confirms that such increases in conditional probability are unlikely to have happened by chance sampling.

Figure 4 suggests that the wavenumber- 5 pattern, through its influence on the likelihood of US heat waves, may benefit probability forecasts of these extreme events on subseasonal timescales. This conclusion is based on the behaviour of a GCM, but its applicability to nature is bolstered by the fact that the wavenumber- 5 pattern is also a prominent subseasonal variability pattern in nature. Thus understanding the origins of this pattern is important. In particular, it needs to be recognized that this pattern can be generated solely by internal dynamics rather than being a response to Asian monsoon rainfall or SSTs. Hence accurate predictions of the monsoon or SSTs may not guarantee subseasonal predictions of US heat waves. However, our study does not preclude the possibility, as the work of others has suggested, that heat waves can also be stimulated by SST anomalies ${ }^{23}$ or land surface conditions ${ }^{24}$.

\section{Methods}

Model and experiment. The 12,000-year atmospheric GCM simulation was conducted with the CAM3 coupled with the Community Land Model, which are the atmosphere and land components of the comprehensive atmosphere-ocean-land-sea-ice fully coupled climate model known as the Community Climate System Model version 3 (ref. 25). We ran CAM3 at a T42 horizontal resolution, which corresponds to approximately a $2.8^{\circ}$ latitude/longitude grid spacing. Monthly varying SST and sea-ice extent, as well as external forcing (solar, anthropogenic), were specified to their present-day climatological values. Although planetary waves produced by CAM3 have a bias in their relationship between frequency and zonal wavenumber (Supplementary Fig. 2), we find in results not described here a similar wavenumber- 5 pattern leading the heat waves in a simulation produced by the latest, fully coupled version of the mode ${ }^{26}$, which simulates a more realistic wavenumber-frequency relationship.
Measure of the heat wave statistics. Based on our definition, there are 16,199 heat wave days from 5,949 events in the 12,000-year simulation and 27 heat wave days from 17 events in the reanalysis during the period of 1948-2012. Heat wave intensity is represented by a composite of SAT anomalies on all heat wave days. To reveal the locations of the heat waves, we define heat wave frequency at a grid point as the number of occurrences of SAT exceeding the threshold value on a heat wave day divided by the total number of heat wave days. The life span of a heat wave event equals of the number of consecutive heat wave days plus 4 .

Significance test. Two significance tests were conducted for the wavenumber-5 $300 \mathrm{hPa}$ streamfunction composite precursor patterns in Fig. 2. One is the standard one-sample Student $t$-test (Supplementary Fig. 4) and the other is a field significance test (Supplementary Fig. 5). For the latter, we employed a Monte Carlo technique in which we calculated averages of randomly drawn daily maps using the same sample size as in Fig. 2. Of the Fig. 2 composites the day -20 composite has the smallest area that passed the $t$-test. Of the 10,000 randomly generated composites only four had larger area that passed the test than the day -20 composite. Hence the fields in Fig. 2 composites are highly significant.

To test the significance of the prediction results in Fig. 4 we have also conducted a Monte-Carlo-based test. For this test the null hypothesis is that increasing conditional probability of heat waves with respect to pattern amplitude (Fig. 4) can also be found for patterns with arbitrary structure. One thousand random patterns are generated from random combinations of 30 EOFs that are derived from $300 \mathrm{hPa}$ streamfunction subseasonal variability from which variability associated with the wavenumber- 5 precursor pattern has been removed. As shown in Fig. 4, we find that the probability of heat waves is much more strongly associated with the amplitude of the precursor pattern than with amplitudes of these random patterns. We therefore reject the null hypothesis.

Results in Fig. 4 remain valid if we apply a cross-validation method, namely, we use half of the 12,000-year data to construct the heat wave composite, then apply it to the other half of the data for finding the likelihood of a heat wave as a function of the amplitude of this pattern.

Received 1 March 2013; accepted 18 September 2013; published online 27 October 2013

\section{References}

1. Easterling, D. R. et al. Climate extremes: Observations, modelling, and impacts. Science 289, 2068-2074 (2000).

2. Ebi, K. \& Meehl, G. A. Heatwaves and Global Climate Change, The Heat is On Climate Change and Heatwaves in the Midwest. Regional Impacts of Climate Change: Four Case Studies in the United States 8-21 (Pew Center on Global Climate Change, 2007).

3. Meehl, G. A. \& Tebaldi, C. More intense, more frequent, and longer lasting heat waves in the 21st century. Science 305, 994-997 (2004).

4. Madden, R. A. \& Julian, P. R. Detection of a 40-50 day oscillation in the zonal wind in the tropical Pacific. J. Atmos. Sci. 28, 702-708 (1971).

5. Waliser, D. E., Lau, K. M., Stern, W. \& Jones, C. Potential predictability of the Madden-Julian oscillation. Bull. Am. Meteorol. Soc. 84, 33-50 (2003).

6. Branstator, G., Mai, A. \& Baumhefner, D. Identification of highly predictable flow elements for spatial filtering of medium- and extended-range numerical forecasts. Mon. Weath. Rev. 121, 1786-1802 (1993).

7. DelSole, T. \& Tippett, M. K. Predictable components and singular vectors. J. Atmos. Sci. 65, 1666-1678 (2008).

8. Schubert, S., Wang, H. \& Suarez, M. Warm seasonal subseasonal variability and climate extremes in the Northern Hemisphere: the role of stationary Rossby waves. J. Clim. 24, 4773-4792 (2011).

9. Chen, P. \& Newman, M. Rossby wave propagation and the rapid development of upper-level anomalous anticyclones during the 1988 US drought. J. Clim. 11, 2491-2504 (1998).

10. Lyon, B. \& Dole, R. M. A diagnostic comparison of the 1980 and 1988 US summer heat wave-droughts. J. Clim. 8, 1658-1675 (1995).

11. Kalnay, E. et al. The NCEP/NCAR 40-year reanalysis project. Bull. Am. Meteorol. Soc. 77, 437-471 (1996).

12. Branstator, G. Horizontal energy propagation in a barotropic atmosphere with meridional and zonal structure. J. Atmos. Sci. 40, 1689-1708 (1983).

13. Hoskins, B. J. \& Ambrizzi, T. Rossby wave propagation on a realistic longitudinally varying flow. J. Atmos. Sci. 50, 1661-1671 (1993).

14. Namias, J. Anatomy of Great Plains protracted heat waves (especially the 1980 US summer drought). Mon. Weath. Rev. 110, 824-838 (1982).

15. Chang, F.-C. \& Wallace, J. M. Meteorological conditions during heat waves and droughts in the United States Great Plains. Mon. Weath. Rev. 115, 1253-1269 (1987).

16. Plumb, R. A. On the three-dimensional propagation of stationary waves. J. Atmos. Sci. 42, 217-229 (1985).

17. Karoly, D. J., Plumb, R. A. \& Ting, M. Example of the horizontal propagation of quasi-stationary waves. J. Atmos. Sci. 46, 2802-2811 (1989). 
18. Hoskins, B. \& Karoly, D. J. The steady linear response of a spherical atmosphere to thermal and orographic forcing. J. Atmos. Sci. 38, 1179-1196 (1981).

19. Branstator, G. Organization of storm track anomalies by recurring low-frequency circulation anomalies. J. Atmos. Sci. 52, 207-226 (1995).

20. Branstator, G. Circumglobal teleconnections, the jet stream waveguide, and the North Atlantic Oscillation. J. Clim. 15, 1893-1910 (2002).

21. Ding, Q. \& Wang, B. Circumglobal teleconnection in the Northern Hemisphere summer. J. Clim. 18, 3483-3505 (2005).

22. Ding, Q., Wang, B., Wallace, J. M. \& Branstator, G. Tropical-extratropical teleconnections in boreal summer: Observed interannual variability. J. Clim. 24, 1878-1896 (2011).

23. Newman, M. \& Sardeshmukh, P. D. The impact of the annual cycle on the North Pacific/North American response to remote low-frequency forcing. J. Atmos. Sci. 55, 1336-1353 (1998).

24. Erich, E. et al. Soil moisture-atmosphere interactions during the 2003 European summer heat wave. J. Clim. 20, 5081-5099 (2007).

25. Collins, W. D. et al. The Community Climate System Model version 3 (CCSM3). J. Clim. 19, 2122-2143 (2006).

26. Gent, P. et al. The community climate system model version 4. J. Clim. 24, 4973-4991 (2011).

\section{Acknowledgements}

We thank A. Mai for carrying out the experiment and S. Schubert for comments. Portion of this study were supported by the Office of Science (BER), US Department of Energy, NASA NEWS and the National Science Foundation. NCAR is sponsored by the National Science Foundation. Computing resources were provided by NCAR's Computational and Information Systems Laboratory and by the National Energy Research Scientific Computing Center, which is supported by BER of the US Department of Energy.

\section{Author contributions}

H.T. and G.B. led the analysis and all authors contributed to writing of the paper.

\section{Additional information}

Supplementary information is available in the online version of the paper. Reprints and permissions information is available online at www.nature.com/reprints. Correspondence and requests for materials should be addressed to H.T.

\section{Competing financial interests}

The authors declare no competing financial interests. 